OPEN ACCESS

Edited by:

Xiaotian Wang

Southwest University, China

Reviewed by:

Jiangchao Han,

Beihang University, China

Xiaoming Zhang,

Hebei University of Technology, China

${ }^{*}$ Correspondence:

Heju Xu

xuheju@ncst.edu.cn

Specialty section: This article was submitted to

Theoretical and Computational

Chemistry,

a section of the journal

Frontiers in Chemistry

Received: 22 September 2020

Accepted: 12 October 2020

Published: 05 November 2020

Citation:

Xu H (2020) Realization of Opened and Closed Nodal Lines and Fourand Three-fold Degenerate Nodal

Points in XPt $(X=S c, Y, L a)$

Intermetallic Compound: $A$

Computational Modeling Study.

Front. Chem. 8:609118.

doi: 10.3389/fchem.2020.609118

\section{Realization of Opened and Closed Nodal Lines and Four- and Three-fold Degenerate Nodal Points in XPt $(X=$ Sc, Y, La) Intermetallic Compound: A Computational Modeling Study}

Heju $X u^{*}$

College of Science, North China University of Science and Technology, Tangshan, China

Realizing rich topological elements in topological materials has attracted increasing attention in the fields of chemistry, physics, and materials science. Topological semimetals/metals are classified into three main types: nodal-point, nodal-line, and nodal-surface types with zero-, one-, and two-dimensional topological elements, respectively. This study reports that XPt $(X=S c, Y, L a)$ intermetallic compounds are topological metals with opened and closed nodal lines, and triply degenerate nodal points (TNPS) when the spin-orbit coupling (SOC) is ignored. Based on the calculated phonon dispersions, one can find that ScPt and YPt are dynamically stable whereas LaPt is not. When SOC is added, the one-dimensional nodal line and zero-dimensional TNPs disappear. Interestingly, a new zero-dimensional topological element, that is, Dirac points with 4-fold degenerate isolated band crossings with linear band dispersion appear. The proposed materials can be considered a good platform to realize zero- and one-dimensional topological elements in a single compound and to study the relationship between zero- and one-dimensional topological elements.

Keywords: 4-fold degenerate nodal point, triply degenerate nodal point (TNP), spin-orbit coupling (SOC), topological element, phonon dispersion

\section{INTRODUCTION}

In the last decade, with the discovery of topological insulators (Cava et al., 2013; Kou et al., 2013; Zhao et al., 2013; Shen and Cha, 2014; Wang et al., 2014; Luo et al., 2015; Zhou et al., 2015; Liu et al., 2016; Chen et al., 2017a; Loïc and Izmaylov, 2017; Pan et al., 2017; Pielnhofer et al., 2017; Politano et al., 2017; Andrey et al., 2018; Hu et al., 2018, 2019; Gao et al., 2019; Mal et al., 2019; Qiao et al., 2019; Narimani et al., 2020), topologically non-trivial materials have attracted significant interest in the chemistry, physics, and materials science communities. Recently, studies have increasingly focused on topological semimetals/metals (Bin et al., 2018; Chenguang et al., 2018; Zhou et al., 2018; He et al., 2019, 2020; Jin et al., 2019a, 2020b; Li et al., 2019; Qie et al., 2019; Xie et al., 2019; Yi et al., 2019; Zhong et al., 2019; Ma and Sun, 2020; Meng et al., 2020b; Wang et al., 2020a,c,d; Yang and Zhang, 2020; Zhang et al., 2020; Zhao et al., 2020) with non-trivial band topology. For example, in 2018, Schoop et al. (2018) described the key features of the electronic structures of topological semimetals/metals and how these structures can be realized based on chemical principles. 
Topological semimetals/metals can be roughly classified into three main parts: nodal-point (Chen et al., 2015; Yuan et al., 2017; Zhang et al., 2017a,c, 2018c; Jing and Heine, 2018; Ma et al., 2018; Tsipas et al., 2018; Khoury et al., 2019), nodal-line (Chang et al., 2016; Liu et al., 2018b; Guo et al., 2019; Sankar et al., 2019; Tang et al., 2019; Xu et al., 2019; Zhang et al., 2019; Jin et al., 2020a; Kirby et al., 2020; Zhou et al., 2020), and nodal-surface (Türker and Sergej, 2018; Wu et al., 2018; Zhang et al., 2018b,d; Fu et al., 2019; Yang et al., 2019b, 2020; Chen et al., 2020; Wang et al., 2020e; Xiao et al., 2020) semimetals/metals enjoying zero-, one-, and two-dimensional topological elements, respectively. The main examples of nodal-point semimetals/metals are Weyl and Dirac semimetals/metals with 2- and 4-fold degenerate band-crossing points with linear dispersion. In addition, 3-, 6-, and 8-fold (Cano and Vergniory, 2016; Lu et al., 2016; Weng et al., 2016b) band degenerates also exist. Among them, nodalpoint semimetals/metals with 3 -fold band degenerates [i.e., triply degenerate nodal point (TNP)] are of importance owing to their special properties. Many investigations have been conducted to predict and confirm new TNP semimetals/metals (Weng et al., 2016a; Xia and Li, 2017; Zhang et al., 2017b,d; Guo et al., 2018; Owerre, 2018; Jin et al., 2019b; Yang et al., 2019a). For example, in 2019, Jin et al. (2019b) reported that centrosymmetric $\mathrm{Li}_{2} \mathrm{NaN}$ is a topological material with critical-type TNPs. A critical-type TNP is an interesting topological metal phase that lies between type-I and type-II TNPs. In 2018, Guo et al. (2018) proposed that $\mathrm{YRh}_{6} \mathrm{Ge}_{4}, \mathrm{LaRh}_{6} \mathrm{Ge}_{4}$, and $\mathrm{LuRh}_{6} \mathrm{Ge}_{4}$ are TNP materials, and what is more, Zhu et al. (2020) performed transport measurements and confirmed TNP fermions in $\mathrm{YRh}_{6} \mathrm{Ge}_{4}$. In 2019, Yang et al. (2019a) experimentally demonstrated TNP as well as double Fermi arc surface states in a three-dimensional phononic crystal.

Nodal-line semimetals/metals with one-dimensional topological elements may show various forms according to the shape of the nodal lines, such as nodal link (Yan et al., 2017), nodal chain (Yan et al., 2018), nodal box (Sheng et al., 2017), nodal ring (Zhang et al., 2018a; Wang et al., 2020b), nodal knot (Bi et al., 2017; Lee et al., 2018), and nodal net (Feng et al., 2018). For example, in 2018, Zhou et al. (2018) proposed that two-dimensional $\mathrm{B}_{2} \mathrm{C}$ hosts opened and closed nodal-line states based on first-principles calculation. In 2020, Yi et al. (2019) predicted that NaAlGe and NaAlSi nodal-line materials would be good cathode materials for sodium ion batteries. In 2020, Wang et al. (2020d) proposed that a two-dimensional $\mathrm{Nb}_{3} \mathrm{GeTe}_{6}$ monolayer is a topological nodal-line material with a nearly flat nodal line around the Fermi level and that it led to a remarkable thermoelectric power factor platform. In 2018, Liu et al. (2018a) proposed that graphene monolith, a three-dimensional nodalline semimetal, is a candidate lithium ion battery anode material. In 2019, Yan et al. (2019) proposed that the $\mathrm{Cu}_{2} \mathrm{Si}$ monolayer is a topological material with possible superconductivity and nodal-line fermions.

The electronic structure, dynamical stability, and topological signatures of ScPt, YPt, and LaPt, a cubic-type family of materials with $\mathrm{Pm} \overline{3} \mathrm{~m}$ space group are investigated in the present study. This study shows that opened and closed nodal lines and 3fold degenerate nodal-point states can be realized in ScPt, LaPt, and LuPt when the spin-orbit coupling is ignored. Moreover, the effect of spin-orbit coupling on the topological signatures of these systems is also considered. A 3- to 4-fold degenerate nodalpoint transition can be found in these systems when spin-orbit coupling is added.

\section{MATERIALS}

The topological signatures of cubic-type $\mathrm{ScPt}$, YPt, and LaPt are investigated. As an example, Figure 1A shows the structural model of cubic ScPt. This primitive ScPt cell contains one Sc atom and one Pt atom at the $(0.5,0.5,0.5)$ and $(0,0,0)$ sites, respectively. Using first-principles calculation, the structural models of ScPt, YPt, and LaPt are fully optimized; Table 1 lists the calculated results.

The phonon dispersions of cubic-type ScPt, YPt, and LaPt are calculated using the force-constants method with Phonopy code (Togo and Tanaka, 2015). For these three compounds, $2 \times 2 \times$ 2 supercells are built to calculate the phonon dispersions. The considered high-symmetry points are $\Gamma-\mathrm{X}-\mathrm{M}-\Gamma-\mathrm{R}-\mathrm{X}$, as shown in Figure 1B. Figures 1C, 2A exhibit the calculated phonon dispersions of ScPt and YPt; one can find that ScPt and YPt are dynamically stable owing to the absence of the imaginary frequency (Han et al., 2019; Wu et al., 2019). However, the obtained phonon dispersion shown in Figure 2B indicates that LaPt is not dynamically stable.

\section{COMPUTATIONAL METHODS}

In this study, first-principles calculations are used, and the generalized gradient approximation (GGA) (Perdew et al., 1996) of the Perdew-Burke-Ernzerhof (PBE) (Perdew et al., 1998) functional is adopted for the exchange-correlation potential. In the calculations, the cutoff energy is set as $600 \mathrm{eV}$, and the Brillouin zone is sampled using a Monkhorst-Pack $k$-mesh with a size of $9 \times 9 \times 9$. To ensure good convergence, the calculations continue until the energy deviation is $<10^{-6} \mathrm{eV} /$ atom. The atomic positions and lattice constants of the structures were totally relaxed until all the force components were smaller than $10^{-3} \mathrm{eV} / \AA$.

\section{RESULTS AND DISCUSSION}

First, the physical natures of ScPt, YPt, and LaPt are determined. Figures 1D, 3A,C, respectively, show the band structures of $\mathrm{ScPt}$, YPt, and LaPt along the R-X-M-R- $\Gamma$ paths. The spin-orbit coupling (SOC) is not added for the band structures in these figures; the effect of SOC on the electronic structures of these compounds will be discussed later in this paper. These three figures show that the bands and the Fermi level overlap each other, indicating common metallic behaviors.

Moreover, some obvious band crossings are seen around the Fermi level, namely, point $\mathrm{A}$ along the R-X path, point $\mathrm{B}$ along the $\mathrm{X}-\mathrm{M}$ path, and point $\mathrm{C}$ along the $\mathrm{M}-\mathrm{R}$ path. A careful study of these three band-crossing points indicates that points $\mathrm{A}$ and $\mathrm{B}$ are doubly degenerate band-crossing points, 
A

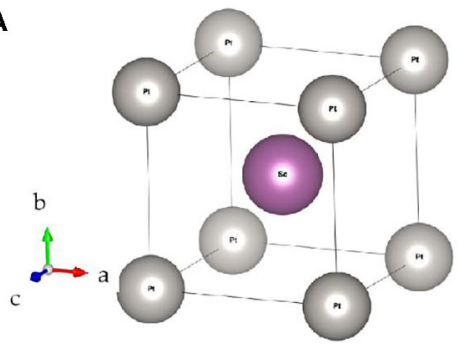

D

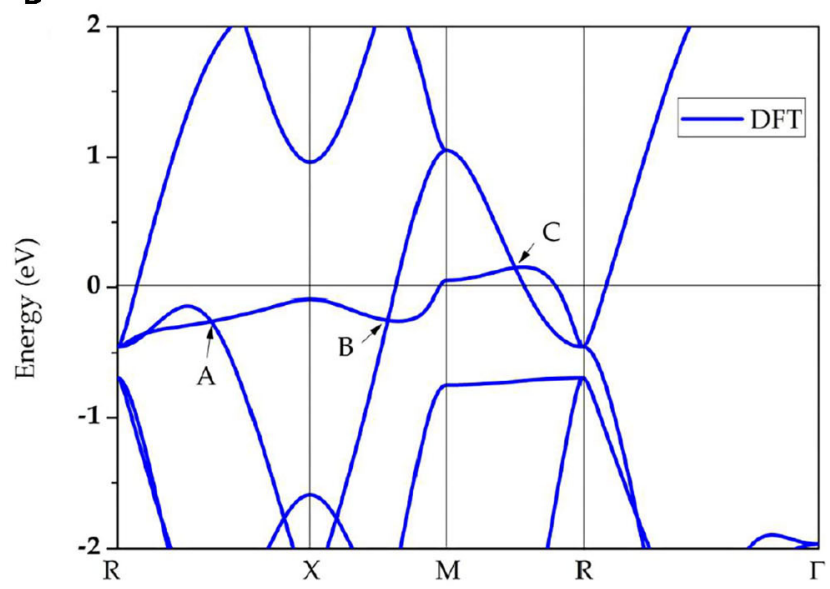

C

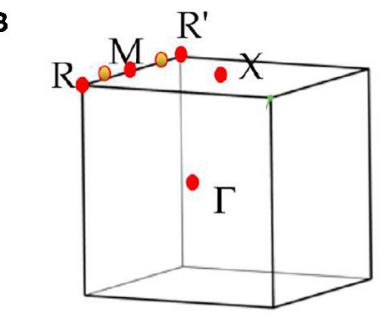

E
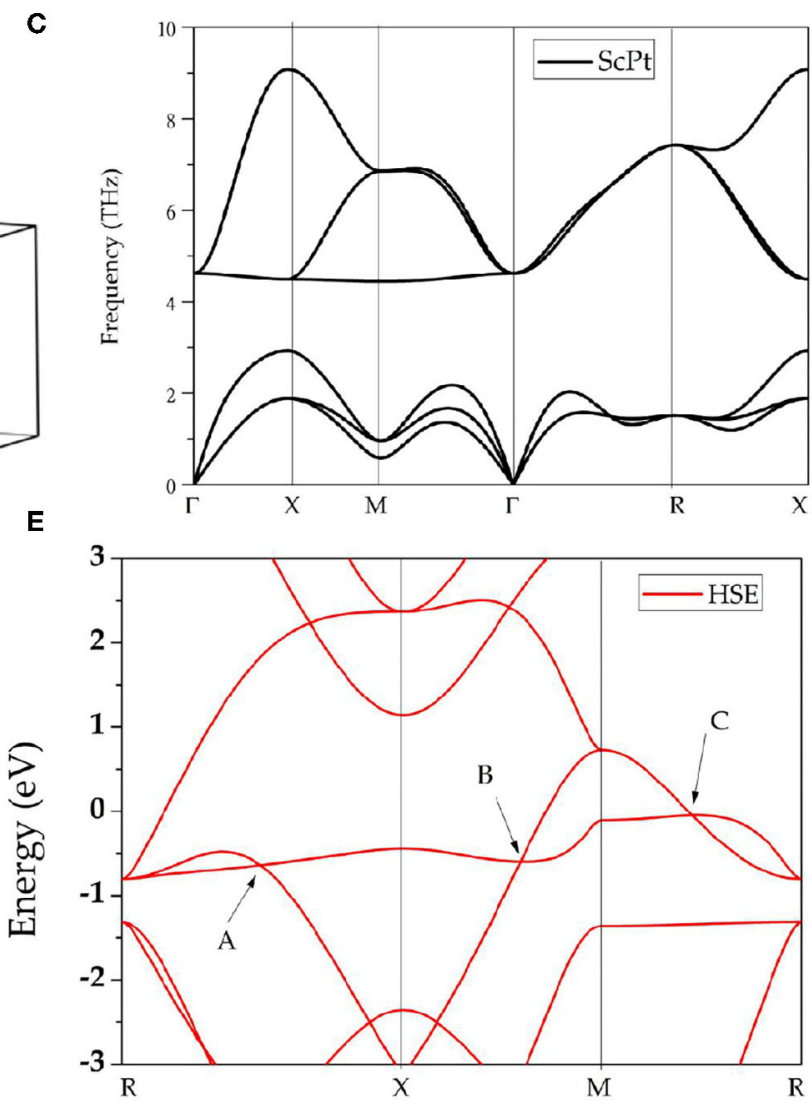

FIGURE 1 | (A) Crystal structures of cubic ScPt; (B) Brillouin zone and the considered high-symmetry points $\Gamma$-X-M- $\Gamma$-R-X; (C) calculated phonon dispersion of cubic ScPt at its optimized lattice constant; (D) calculated band structure of cubic ScPt with PBE method, where (A-C) indicate the band-crossing points around the Fermi level; and (E) calculated band structure of ScPt with Heyd-Scuseria-Ernzerhof (HSE) screened hybrid functional.

TABLE 1 | Optimized lattice constants for ScPt, YPt, and LaPt.

\begin{tabular}{llll}
\hline Compounds & $\mathbf{a}(\mathbf{\AA})$ & $\mathbf{b}(\AA)$ & $\mathbf{c}(\AA)$ \\
\hline $\mathrm{ScPt}$ & 3.283 & 3.283 & 3.283 \\
$\mathrm{YPt}$ & 3.488 & 3.488 & 3.488 \\
$\mathrm{LaPt}$ & 3.659 & 3.659 & 3.659 \\
\hline
\end{tabular}

whereas point $\mathrm{C}$ is a 3 -fold degenerate band-crossing point formed by a doubly degenerate band and a non-degenerate band. Apart from these three clear band-crossing points, the band structure near the Fermi level is very clean; therefore, these three points dominate the topological signatures of these compounds. For clarity, hereafter, ScPt is used as an example to investigate the band topology considering that the band structures of ScPt, YPt, and LaPt are almost the same near the Fermi level.

Figure 1E shows the band structure of ScPt with the revised Heyd-Scuseria-Ernzerhof (HSE) (Heyd and Scuseria, 2004) screened hybrid functional. The HSE method is well-known to be accurate for describing the band gap of topological materials. In particular, for some $d$-orbital-dominated systems, the GGA method cannot provide a fair evaluation of the band gap around the Fermi level. Figures 1D,E show that the band-crossing points $\mathrm{A}, \mathrm{B}$, and $\mathrm{C}$ are still maintained under the HSE method; this confirms that the GGA method is suitable for investigating the electronic structure of the ScPt system.

In addition to SOC, the ScPt system enjoys time reversal $(T)$ and spatial inversion $(P)$ symmetries. Basically, doubly degenerate band crossings like points $\mathrm{A}$ and $\mathrm{B}$ should not be isolated (Weng et al., 2016b; Zhang et al., 2018b). Instead, they should belong to one type of nodal structure; they most commonly belong to a nodal-line structure. As shown in Figures 1D, 3A,C, two bands cross each other and form two band-crossing points $\mathrm{A}$ and $\mathrm{B}$ along the $\mathrm{R}-\mathrm{X}$ and $\mathrm{X}$ $M$ paths. Symmetry analysis shows that these two bands belong to irreducible representations $A_{1 g}$ and $A_{2 u}$ of $D_{4 h}$ symmetry, respectively.

Figure 4A shows the $\mathrm{X}$-centered $3 \mathrm{D}$ band dispersion of the $\mathrm{k}_{\mathrm{z}}$ $=\pi$ plane. The two above-mentioned bands form a closed nodal line in the $\mathrm{k}_{\mathrm{z}}=\pi$ plane (highlighted by a white line), and points $\mathrm{A}$ and $\mathrm{B}$ belong to this closed nodal line. The crystal symmetry of the ScPt cubic system implies three closed nodal lines in the $\mathrm{k}_{\mathrm{x} / \mathrm{y} / \mathrm{z}}=\pi$ planes. The nodal lines are located in the mirrorinvariant plane, and they protect the mirror symmetry $\mathrm{M}_{\mathrm{x}, \mathrm{y}, \mathrm{z}}$. As 
A

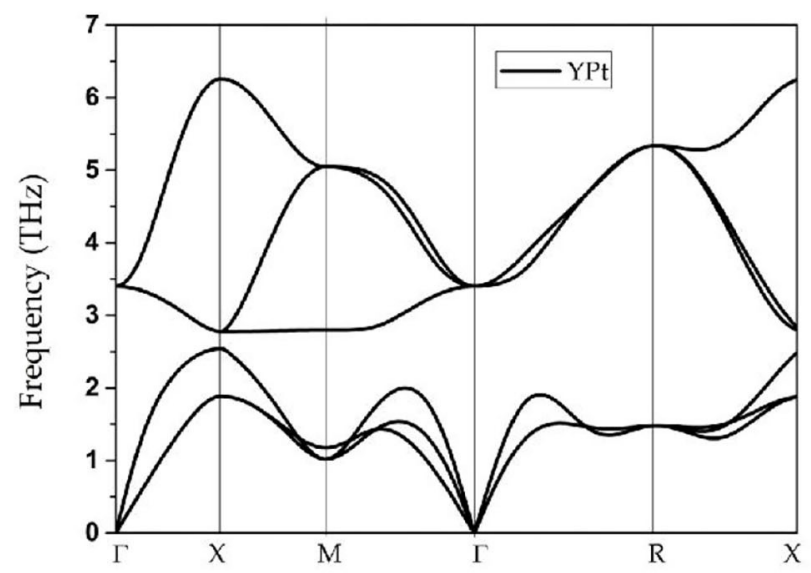

B

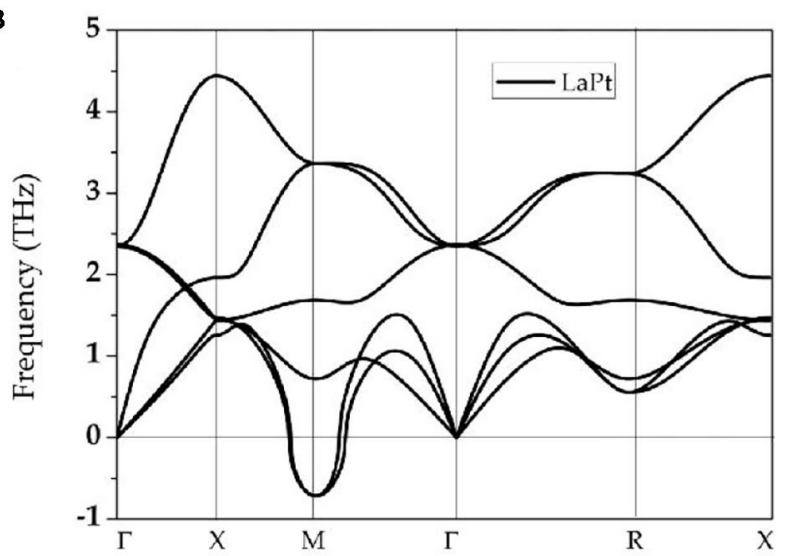

FIGURE 2 | (A,B) Calculated phonon dispersion of cubic YPt and LaPt, respectively, at their optimized lattice constants. The phonon dispersions of both intermetallic compounds are obtained using the force-constants method with Phonopy code.

A

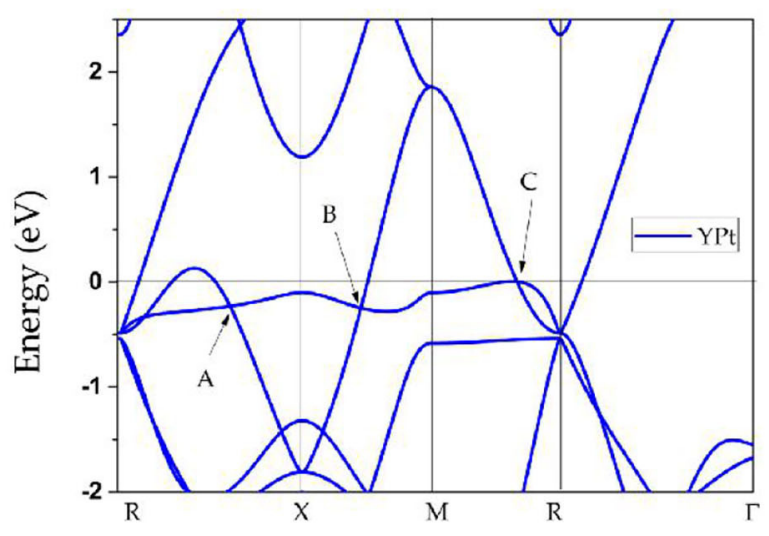

C

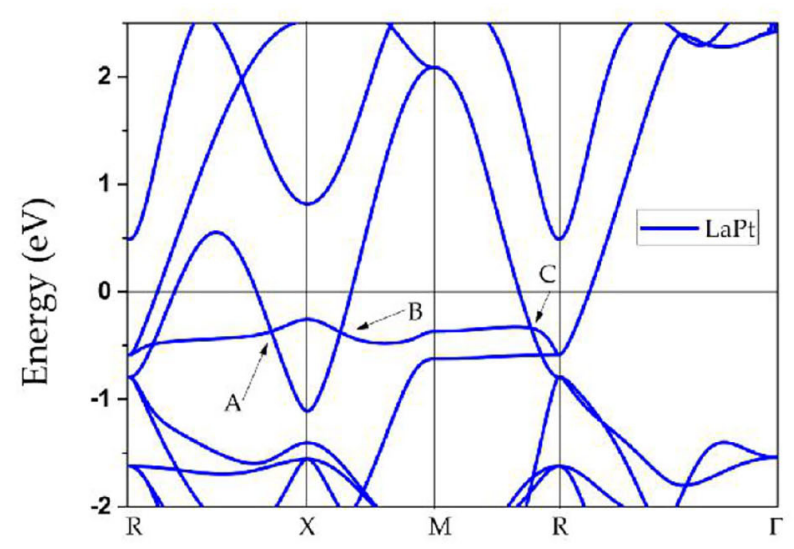

B

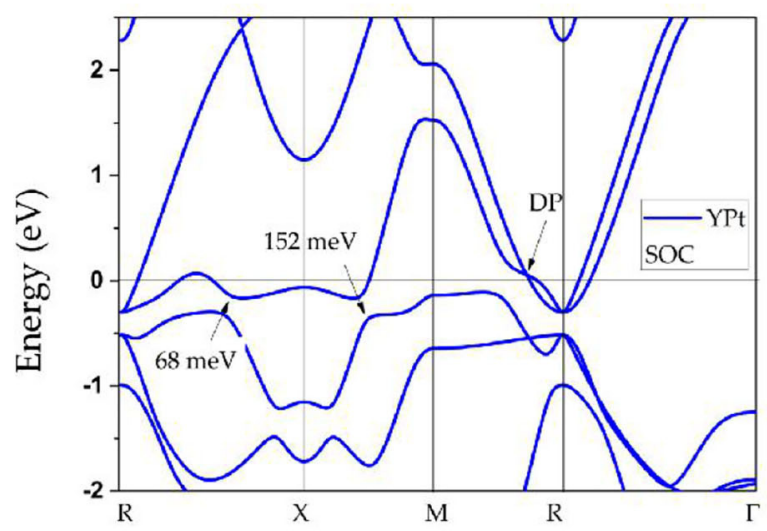

D

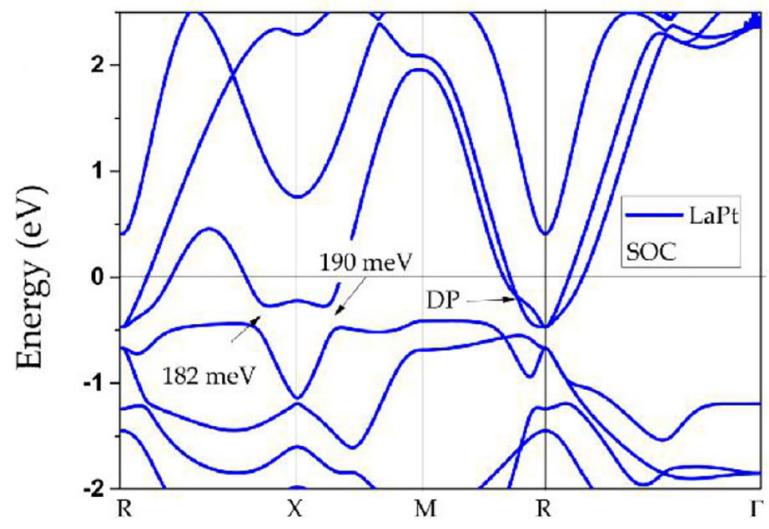

FIGURE 3 | (A-D) Calculated band structures of cubic YPt and LaPt with their optimized lattice constants. The spin-orbit coupling effect is neglected for (A,C) and added for $(\mathbf{B}, \mathbf{D})$.

an example, Figure 4B shows the shape of the $\mathrm{X}$-centered closed nodal line in the $\mathrm{k}_{\mathrm{z}}=\pi$ plane.

Figure $4 \mathrm{C}$ shows the R-centered 3D band dispersion in the $\mathrm{k}_{\mathrm{z}}$ $=\pi$ plane; here, TNPs are indicated by green balls. As shown in
Figures 1D, 3A,C, the band-crossing point $C$ is formed by a 2fold degenerate band and a non-degenerate band along the $\mathrm{M}-\mathrm{R}$ path. This 2-fold degenerate band can be seen as two independent bands that are completely degenerated along the whole M-R path. 


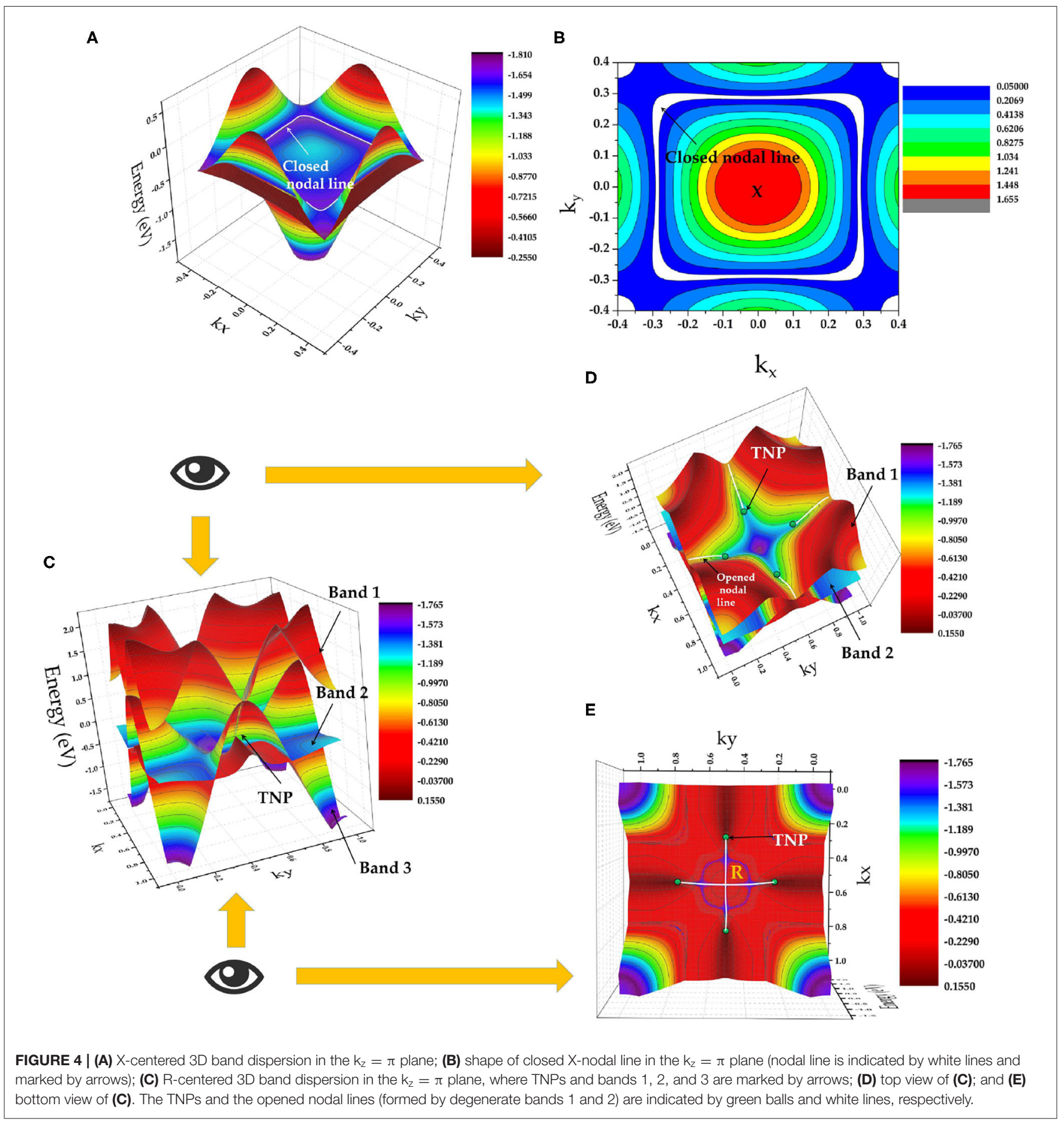

Therefore, this 2-fold degenerate band (named as band 1 and band 2) should contain a series of band-crossing points along the whole M-R path and form an opened nodal line along the M-R path. To clearly present the opened nodal lines and the TNPs in ScPt, Figures 4D,E, respectively, show top and bottom views of Figure 4C. These figures clearly show the opened nodal lines formed from bands 1 and 2 along the R-M path as well as the TNPs. Therefore, ScPt, YPt, and LaPt are topological metals that co-exhibit opened and closed nodal lines when the spinorbit coupling is ignored. Based on the above-mentioned results, the ScPt family of materials is a good platform to study the relationship of closed and opened nodal lines.

Moreover, band-crossing point $\mathrm{C}$ along the $\mathrm{M}-\mathrm{R}$ path is a TNP. Normally, TNP can not only occur in isolation but also 

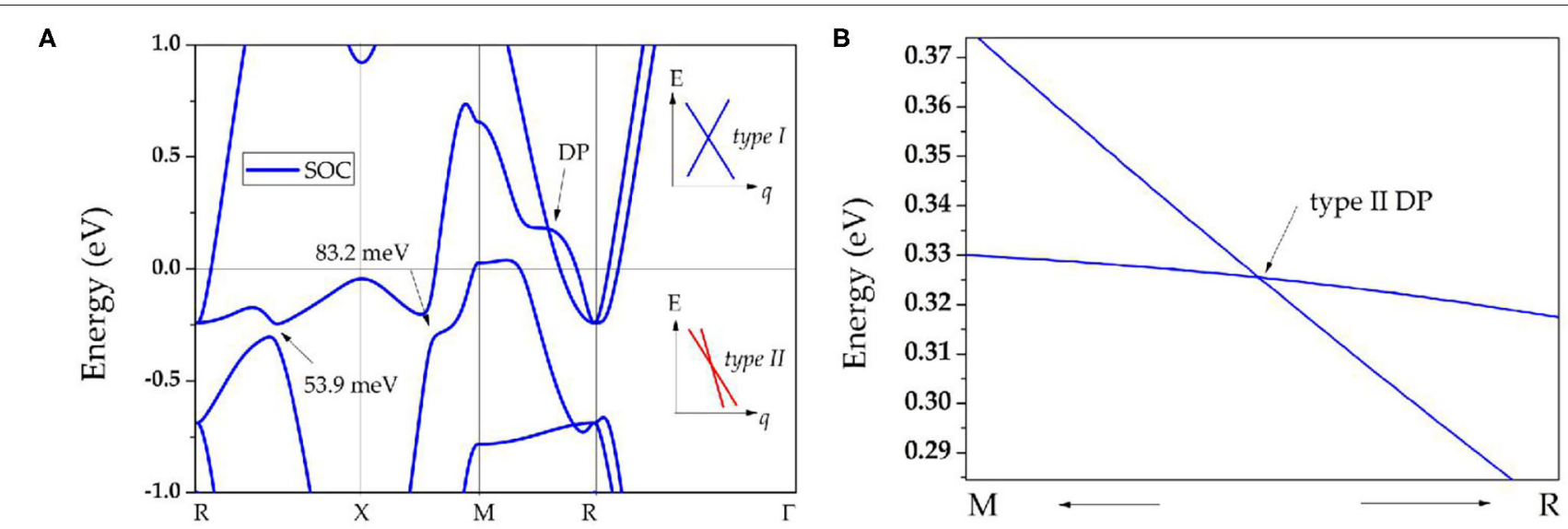

FIGURE 5 | (A) Band structure of ScPt with spin-orbit coupling; the insets show schematics of types I and II nodal points, indicated by blue and red lines, respectively; for type I nodal points, they are conventional type band dispersion, however, for type II nodal points, they enjoy a tilted band dispersion; (B) enlarged band structure of ScPt along the M-R path slightly above the Fermi level.

be linked by nodal lines in the momentum space. One pair of TNPs exists in ScPt, YPt, and LaPt. Figure 1B shows a schematic of the pair of TNPs (indicated by yellow balls) along the R-M$\mathrm{R}^{\prime}$ path. As shown in Figure 4C, the TNPs are located at the crossing of band 3 (non-degenerate band) and bands 1 and 2 (2fold degenerate band). Therefore, $\mathrm{ScPt}$ is concluded to have onedimensional topological elements, namely, opened and closed nodal lines (in the $\mathrm{k}_{\mathrm{x} / \mathrm{y} / \mathrm{z}}=\pi$ plane) and a zero-dimensional topological element, namely, TNP, along the R-M-R' path when the spin-orbit coupling is ignored. Therefore, $\mathrm{ScPt}$, LaPt, and YPt are excellent target materials for studying the entanglement between nodal-line and nodal-point fermions.

Finally, the effect of SOC on the band structures of ScPt, YPt, and LaPt is investigated. The corresponding results are shown in Figures 5A, 3B,D, respectively. The gaps induced by SOC for band-crossing points $\mathrm{A}$ and $\mathrm{B}$ are 53.9 and $83.2 \mathrm{meV}, 68$ and 152 $\mathrm{meV}$, and 182 and $190 \mathrm{meV}$ for ScPt, YPt, and LaPt, respectively. In comparison, the gaps induced by SOC in the well-known nodal-line materials $\mathrm{Cu}_{2} \mathrm{NPd}$ (Yu et al., 2015), CaAgBi (Chen et al., 2017b), and $\mathrm{BaSn}_{2}$ (Huang et al., 2016) are 60-100 meV, 80-140 meV, and 60-160 meV, respectively. Therefore, ScPt and YPt are comparable to these reference materials.

Moreover, Figures 5B, 3B,D show that the TNPs disappear in $\mathrm{ScPt}, \mathrm{YPt}$, and LaPt systems. However, a new topological signature reveals a nodal point with linear band dispersion around the Fermi level. When the SOC effect was considered, each band was doubly degenerate. Therefore, the newly occurring nodal point along the along R-M path should be a Dirac nodal point (DP) with 4-fold degeneracy. Specifically, a pair of DPs with 4-fold degeneracy is found along the R-M-R' path. Notably, similar SOC-induced TNP-DP transitions have also been reported in ErAs (Meng et al., 2020a), TiB 2 (Zhang et al., $2017 \mathrm{~d}$ ), and $\mathrm{Li}_{2} \mathrm{NaN}$ (Jin et al., 2019b) topological materials.
However, unlike the type-I DP predicted for ErAs, this is a type-II DP that may show strong anisotropy (Zhang et al., 2018b).

\section{SUMMARY}

In summary, cubic-type $\mathrm{ScPt}$, YPt, and LaPt are shown to be newly designed topological materials through the use of density functional theory. ScPt and YPt are dynamically stable whereas LaPt is not. Without SOC, XPt (X = Sc, Y, La) metals show closed and opened nodal-line states and one pair of TNPs. With SOC, the TNPs (along the R-M-R' path) change to type-II DPs and the nodal-line states in $\mathrm{k}_{\mathrm{x} / \mathrm{y} / \mathrm{z}}=\pi$ planes are gapped. A series of interesting topological signatures has been predicted in XPt ( $\mathrm{X}=\mathrm{Sc}, \mathrm{Y}, \mathrm{La}$ ), and it is hoped that these proposed topological elements can be confirmed through experiments in the future.

\section{DATA AVAILABILITY STATEMENT}

The original contributions presented in the study are included in the article/supplementary materials, further inquiries can be directed to the corresponding author/s.

\section{AUTHOR CONTRIBUTIONS}

The author confirms being the sole contributor of this work and has approved it for publication.

\section{FUNDING}

This work was funded by the Science and Technology Research Project of Hebei Province Colleges and Universities (Grant No. QN2020113) and Tangshan Applied Basic Research Project (Grant No. 19130227g). 


\section{REFERENCES}

Andrey, V., Sánchez-Barriga, J., Maria, B., Carolien, C., Joke, H., and Anna, S. (2018). Can surface reactivity of mixed crystals be predicted from their counterparts? A case study of $\left(\mathrm{Bi}_{1--\mathrm{X}} \mathrm{Sb}_{\mathrm{x}}\right)_{2} \mathrm{Te}_{3}$ topological insulators. J. Mater. Chem. C 6, 8941-8949. doi: 10.1039/C8TC02235F

Bi, R., Yan, Z., Lu, L., and Wang, Z. (2017). Nodal-knot semimetals. Phys. Rev. B 96:201305. doi: 10.1103/PhysRevB.96.201305

Bin, Q., Tang, F., Ruan, Y. R., Yong, F., and Han, Z. D. (2018). Extremely large magnetoresistance in the nonmagnetic semimetal YBi. J. Mater. Chem. C 6, 10020-10029. doi: 10.1039/C8TC02839G

Cano, J., and Vergniory, M. G. (2016). Beyond dirac and Weyl fermions: unconventional quasiparticles in conventional crystals. Science 353:aaf5037. doi: $10.1126 /$ science.aaf5037

Cava, R. J., Ji, H., Fuccillo, M. K., Gibson, Q. D., and Hor, Y. S. (2013). Crystal structure and chemistry of topological insulators. J. Mater. Chem. C 1, 3176-3189. doi: 10.1039/c3tc30186a

Chang, G., Xu, S. Y., Zheng, H., Singh, B., Hsu, C. H., and Bian, G. (2016). Roomtemperature magnetic topological Weyl fermion and nodal line semimetal states in half-metallic Heusler $\mathrm{Co}_{2} \mathrm{TiX}(\mathrm{X}=\mathrm{Si}, \mathrm{Ge}$, or $\mathrm{Sn})$. Sci. Rep. 6:38839. doi: $10.1038 /$ srep 38839

Chen, C., Wang, S. S., Liu, L., Yu, Z. M., and Yang, S. A. (2017b). Ternary wurtzite $\mathrm{CaAgBi}$ materials family: A playground for essential and accidental, type-I and type-II Dirac fermions. Phys. Rev. Mater. 1:044201. doi: 10.1103/PhysRevMaterials.1.044201

Chen, K. X., Lyu, S. S., Luo, Z. Y., Fu, Y. X., Heng, Y., and Mo, D. C. (2017a). Theoretical design of a new family of two-dimensional topological insulators. Phys. Chem. Chem. Phys.19, 7481-7485. doi: 10.1039/C6CP08670E

Chen, S. Z., Li, S., Chen, Y., and Duan, W. (2020). Nodal flexiblesurface semimetals: case of carbon nanotube networks. Nano Lett. 20:5400. doi: 10.1021/acs.nanolett.0c01786

Chen, Z., Zhang, C., Zhou, Y., Zhang, E., Yang, L., Hong, M., et al. (2015). Scalable growth of high mobility dirac semimetal $\mathrm{Cd}_{3} \mathrm{As}_{2}$ microbelts. Nano Lett. 15, 5830-5834. doi: 10.1021/acs.nanolett.5b01885

Chenguang, F., Narayan, G. S., June, W. S., Guowei, L., Enke, L., and Nitesh, K. (2018). Large Nernst power factor over a broad temperature range in polycrystalline Weyl semimetal NbP. Energy Environ. Sci. 11, 2813-2820. doi: 10.1039/C8EE02077A

Feng, X., Yue, C., Song, Z., Wu, Q. S., and Wen, B. (2018). Topological dirac nodal-net fermions in $\mathrm{AlB}_{2}$-type $\mathrm{TiB}_{2}$ and $\mathrm{ZrB}_{2}$. Phys. Rev. Mater. 2:014202. doi: 10.1103/PhysRevMaterials.2.014202

Fu, B.-B., Yi, C., Zhang, T., Caputo, M., Ma, Z., Gao, X., et al. (2019). Dirac nodal surfaces and nodal lines in ZrSiS. Sci. Adv. 5:eaau6459. doi: $10.1126 /$ sciadv.aau6459

Gao, L., Sun, J., Sethi, G., Zhang, Y., Du, S., and Liu, F. (2019). Orbital design of topological insulators from two-dimensional semiconductors. Nanoscale 11, 22743-22747. doi: 10.1039/C9NR06859G

Guo, L., Chen, T. W., Chen, C., Chen, L., Zhang, Y., and Gao, G. Y. (2019). Electronic transport evidence for topological nodal-line semimetals of ZrGeSe single crystals. ACS Appl. Electr. Mater. 1, 869-876. doi: 10.1021/acsaelm.9b00061

Guo, P. J., Yang, H. C., Liu, K., and Lu, Z. Y. (2018). Triply degenerate nodal points in $\mathrm{RRh}_{6} \mathrm{Ge}_{4}(\mathrm{R}=\mathrm{Y}, \mathrm{La}, \mathrm{Lu})$. Phys. Rev. B 98:045134. doi: 10.1103/PhysRevB.98.045134

Han, Y., Wu, M., Feng, Y., Cheng, Z. X., and Wang, X. T. (2019). Competition between cubic and tetragonal phases in all-d-metal Heusler alloys, $\mathrm{X}_{2-\mathrm{x}} \mathrm{Mn}_{1+\mathrm{x}} \mathrm{V}(\mathrm{X}=\mathrm{Pd}, \mathrm{Ni}, \mathrm{Pt}, \mathrm{Ag}, \mathrm{Au}, \mathrm{Ir}, \mathrm{Co} ; \mathrm{x}=1,0)$ : a new potential direction of the Heusler family. IUCrJ 6, 465-472. doi: 10.1107/S20522525190 04007

He, T., Zhang, X., Liu, Y., Dai, X., Liu, G., Liu, Z., et al. (2020). Ferromagnetic hybrid nodal loop and switchable type-I and type-II Weyl fermions in two dimensions. Phys. Rev. B 102:075133. doi: 10.1103/PhysRevB.102.075133

He, T., Zhang, X., Meng, W., Jin, L., Dai, X., and Liu, G. (2019). Topological nodal lines and nodal points in the antiferromagnetic material $\beta-\mathrm{Fe}_{2} \mathrm{PO}_{5}$. J. Mater. Chem. C 7, 12657-12663. doi: 10.1039/C9TC04046C

Heyd, J., and Scuseria, G. (2004). Efficient hybrid density functional calculations in solids: Assessment of the Heyd-Scuseria-Ernzerhof screened Coulomb hybrid functional. J. Chem. Phys. 121, 1187-1192. doi: 10.1063/1.1760074
Hu, X., Pang, Z., Chen, X., Ren, M., and Li, P. (2018). Two-dimensional topological insulators of $\mathrm{Pb} / \mathrm{Sb}$ honeycombs on a Ge (111) semiconductor surface. RSC Adv. 8, 34999-35004. doi: 10.1039/C8RA06316H

Hu, X. K., Pang, Z. X., Zhang, C. W., Wang, P. J., Li, P., and Ji, W. X. (2019). A two-dimensional robust topological insulator with coexisting ferroelectric and valley polarization. J. Mater. Chem. C 7, 9406-9412. doi: 10.1039/C8TC06252H

Huang, H., Liu, J., Vanderbilt, D., and Duan, W. (2016). Topological nodal-line semimetals in alkaline-earth stannides, germanides, and silicides. Phys. Rev. B 93:201114. doi: 10.1103/PhysRevB.93.201114

Jin, L., Zhang, X., Dai, X., Liu, H., Chen, G., and Liu, G. (2019b). Centrosymmetric $\mathrm{Li}_{2} \mathrm{NaN}$ : a superior topological electronic material with critical-type triply degenerate nodal points. J. Mater. Chem. C 7, 1316-1320. doi: 10.1039/C8TC05930F

Jin, L., Zhang, X., He, T., Meng, W., and Liu, G. (2020a). Ferromagnetic twodimensional metal-chlorides $\mathrm{MCl}(\mathrm{M}=\mathrm{Sc}, \mathrm{Y}$, and $\mathrm{La})$ : candidates for Weyl nodal line semimetals with small spin-orbit coupling gaps. Appl. Surf. Sci. 520:146376. doi: 10.1016/j.apsusc.2020.146376

Jin, L., Zhang, X., Liu, Y., Dai, X., Shen, X., and Wang, L. (2020b). Two-dimensional Weyl nodal-line semimetal in a $\mathrm{d}^{0}$ ferromagnetic $\mathrm{K}_{2} \mathrm{~N}$ monolayer with a high Curie temperature. Phys. Rev. B 102:125118. doi: 10.1103/PhysRevB.102.125118

Jin, L., Zhang, X. M., Dai, X. F., Wang, L. Y., and Liu, G. D. (2019a). Screening topological materials with a CsCl-type structure in crystallographic databases. IUCrJ 6, 688-694. doi: 10.1107/S2052252519007383

Jing, Y., and Heine, T. (2018). Two-dimensional kagome lattices made of hetero triangulenes are Dirac semimetals or single-band semiconductors. J. Am. Chem. Soc. 141, 743-747. doi: 10.1021/jacs.8b09900

Khoury, J. F., Rettie, A. J. E., Khan, M. A., Ghimire, N. J., and Kanatzidis, M. G. (2019). A new three-dimensional subsulfide Ir2In8S with Dirac semimetal behavior. J. Am. Chem. Soc. 141, 19130-19137. doi: 10.1021/jacs.9b10147

Kirby, R. J., Ferrenti, A., Weinberg, C., Klemenz, S., and Schoop, L. M. (2020). Transient drude response dominates near-infrared pump-probe reflectivity in nodal-line semimetals ZrSiS and ZrSiSe. J. Phys. Chem. Lett. 11, 6105-6111. doi: 10.1021/acs.jpclett.0c01377

Kou, L., Yan, B., Hu, F., Wu, S. C., Wehling, T. O., and Felser, C. (2013). Graphene-based topological insulator with an intrinsic bulk band gap above room temperature. Nano Lett. 13, 6251-6255. doi: 10.1021/nl4037214

Lee, C. H., Li, G., Liu, Y., Tai, T., Thomale, R., and Zhang, X. (2018). Tidal surface states as fingerprints of non-hermitian nodal knot metals. arXiv [Preprint] arXiv: 1812.02011

Li, G., Xu, Q., Shi, W. J., Fu, C., and Felser, C. (2019). Surface states in bulk single crystal of topological semimetal $\mathrm{Co}_{3} \mathrm{Sn}_{2} \mathrm{~S}_{2}$ toward water oxidation. Sci. Adv. 5:eaaw9867. doi: 10.1126/sciadv.aaw9867

Liu, C., Zhang, H., Sun, Z., Ding, K., and Jie, J. (2016). Topological insulator $\mathrm{Bi}_{2} \mathrm{Se}_{3}$ nanowire/Si heterostructure photodetectors with ultrahigh responsivity and broadband response. J. Mater. Chem. C 4, 5648-5655. doi: 10.1039/C6TC01083K

Liu, J., Li, X., Wang, Q., Kawazoe, Y., and Jena, P. (2018a). A new 3D Dirac nodalline semi-metallic graphene monolith for lithium ion battery anode materials. J. Mater. Chem. A 6, 13816-13824. doi: 10.1039/C8TA04428G

Liu, Z., Xin, H., Fu, L., Liu, Y., Song, T., and Cui, X. (2018b). All-silicon topological semimetals with closed nodal line. J. Phys. Chem. Lett. 10, 244-250. doi: $10.1021 /$ acs.jpclett.8b03345

Loïc, J., and Izmaylov, A. F. (2017). Molecular "topological insulators": a case study of electron transfer in the bis (methylene) adamantyl carbocation. Chem. Commun. 53, 7365-7368. doi: 10.1039/C7CC 02275A

Lu, L., Fang, C., Fu, L., Johnson, S. G., Joannopoulos, J. D., and Solja, I. M. (2016). Symmetry-protected topological photonic crystal in three dimensions. Nat. Phys. 12, 337-340. doi: 10.1038/nphys3611

Luo, L., Zheng, K., Zhang, T., Liu, Y. H., Yu, Y., and Lu, R. (2015). Optoelectronic characteristics of a near infrared light photodetector based on a topological insulator $\mathrm{Sb}_{2} \mathrm{Te}_{3}$ film. J. Mater. Chem. C 3, 9154-9160. doi: 10.1039/C5TC01772F

Ma, H., Chen, P., Li, B., Li, J., Ai, R., Zhang, Z., et al. (2018). Thicknesstunable synthesis of ultrathin type-II Dirac semimetal $\mathrm{PtTe}_{2}$ single crystals and their thickness-dependent electronic properties. Nano Lett. 18, 3523-3529. doi: 10.1021/acs.nanolett.8b00583 
Ma, L., and Sun, Q. (2020). A topological semimetal $\mathrm{Li}_{2} \mathrm{CrN}_{2}$ sheet as a promising hydrogen storage material. Nanoscale 12, 12106-12113. doi: 10.1039/D0NR02180F

Mal, P., Bera, G., Turpu, G. R., Srivastava, S. K., and Das, P. (2019). Vibrational spectra of $\mathrm{Pb}_{2} \mathrm{Bi}_{2} \mathrm{Te}_{3}, \mathrm{PbBi}_{2} \mathrm{Te}_{4}$, and $\mathrm{PbBi}_{4} \mathrm{Te}_{7}$ topological insulators: temperature-dependent Raman and theoretical insights from DFT simulations. Phys. Chem. Chem. Phys. 21, 15030-15039. doi: 10.1039/C9CP01494B

Meng, W., Zhang, X., He, T., Jin, L., and Liu, G. (2020a). Multiple fermionic states with clear nontrivial surface signature in CsCl-type compound ErAs. Comput. Mater. Sci. 183:109815. doi: 10.1016/j.commatsci.2020.109815

Meng, W., Zhang, X., He, T., Jin, L., and Liu, G. (2020b). Ternary compound HfCuP: An excellent Weyl semimetal with the coexistence of type-I and type-II Weyl nodes. J. Adv. Res. 24:523. doi: 10.1016/j.jare.2020.05.026

Narimani, M., Shahram, Y., and Zahra, N. (2020). High thermoelectric efficiency of $\mathrm{LaX}(\mathrm{X}=\mathrm{Sb}, \mathrm{Bi})$ two dimensional topological insulators. J. Phys. Conden. Matter. 32:255501. doi: 10.1088/1361-648X/ab6046

Owerre, S. A. (2018). Magnonic triply-degenerate nodal points. Europhys. Lett. 120:57002. doi: 10.1209/0295-5075/120/57002

Pan, Z., Xue, L., and Sun, L. (2017). Large gap two dimensional topological insulators: the bilayer triangular lattice $\mathrm{TlM}(\mathrm{M}=\mathrm{N}, \mathrm{P}, \mathrm{As}, \mathrm{Sb})$. J. Mater. Chem. C 5, 4268-4274. doi: 10.1039/C7TC00634A

Perdew, J. P., Burke, K., and Ernzerhof, M. (1996). Generalized gradient approximation made simple. Phys. Rev. Lett. 77:3865. doi: 10.1103/PhysRevLett.77.3865

Perdew, J. P., Burke, K., and Ernzerhof, M. (1998). Perdew, burke, and ernzerhof reply. Phys. Rev. Lett. 80:891. doi: 10.1103/PhysRevLett.80.891

Pielnhofer, F., Menshchikova, T. V., Rusinov, I. P., Zeugner, A., Sklyadneva, I. Y., and Heid, R. (2017). Designing 3D topological insulators by 2D-Xene (X = $\mathrm{Ge}, \mathrm{Sn}$ ) sheet functionalization in GaGeTe-type structures. J. Mater. Chem. C 5, 4752-4762. doi: 10.1039/C7TC00390K

Politano, A., Vitiello, M. S., Viti, L., Boukhvalov, D. W., and Chiarello, G. (2017). The role of surface chemical reactivity in the stability of electronic nanodevices based on two-dimensional materials "beyond graphene" and topological insulators. FlatChem 1, 60-64. doi: 10.1016/j.flatc.2016.11.003

Qiao, J., Chuang, M. Y., Lan, J. C., Lin, Y. Y., Sung, W. H., and Fan, R. (2019). Two-photon absorption within layered $\mathrm{Bi}_{2} \mathrm{Te}_{3}$ topological insulators and the role of nonlinear transmittance therein. J. Mater. Chem. C 7, 7027-7034. doi: 10.1039/C9TC01885A

Qie, Y., Liu, J., Wang, S., Sun, Q., and Jena, P. (2019). Tetragonal C24, a topological nodal-surface semimetal with potential as an anode material for sodium ion batteries. J. Mater. Chem. A 7, 5733-5739. doi: 10.1039/C8TA11276B

Sankar, R., Muthuselvam, I. P., Babu, K. R., Murugan, G. S., and Chou, F. C. (2019). Crystal growth and magnetic properties of topological nodal-line semimetal gdsbte with antiferromagnetic spin ordering. Inorg. Chem. 58, 11730-11737. doi: 10.1021/acs.inorgchem.9b01698

Schoop, L. M., Pielnhofer, F., and Lotsch, B. V. (2018). Chemical principles of topological semimetals. Chem. Mater. 30, 3155-3176. doi: 10.1021/acs.chemmater.7b05133

Shen, J., and Cha, J. J. (2014). Topological crystalline insulator nanostructures. Nanoscale 6, 14133-14140. doi: 10.1039/C4NR05124F

Sheng, X. L., Yu, Z. M., Yu, R., Weng, H., and Yang, S. A. (2017). d orbital topological insulator and semimetal in the antifluorite $\mathrm{Cu}_{2} \mathrm{~S}$ family: contrasting spin Helicities, nodal box, and hybrid surface states. J. Phys. Chem. Lett. 8, 3506-3511. doi: 10.1021/acs.jpclett.7b01390

Tang, M., Shen, H., Qie, Y., Xie, H., and Sun, Q. (2019). Edge-state-enhanced $\mathrm{CO}_{2}$ electroreduction on topological nodal-line semimetal $\mathrm{Cu}_{2} \mathrm{Si}$ nanoribbons. J. Phys. Chem. C 123, 2837-2842. doi: 10.1021/acs.jpcc.8b08871

Togo, A., and Tanaka, I. (2015). First principles phonon calculations in materials science. Scr. Mater. 108, 1-5. doi: 10.1016/j.scriptamat.2015.07.021

Tsipas, P., Tsoutsou, D., Fragkos, S., Sant, R., Alvarez, C., and Okuno, H. (2018). Massless dirac fermions in $\mathrm{ZrTe}_{2}$ semimetal grown on InAs (111) by van der Waals epitaxy. ACS Nano 12, 1696-1703. doi: 10.1021/acsnano.7b08350

Türker, O., and Sergej, M. (2018). Weyl nodal surfaces. Phys. Rev. B 97:075120. doi: 10.1103/PhysRevB.97.075120

Wang, A., Zhang, X., and Zhao, M. (2014). Topological insulator states in a honeycomb lattice of s-triazines. Nanoscale 6, 11157-11162. doi: $10.1039 / \mathrm{C} 4 \mathrm{NR} 02707 \mathrm{H}$
Wang, X., Cheng, Z., Zhang, G., Wang, B., Wang, X. L., and Chen, H. (2020c). Rich novel zero-dimensional (0D), 1D, and 2D topological elements predicted in the $\mathrm{P}_{3} / \mathrm{m}$ type ternary boride $\mathrm{HfIr}_{3} \mathrm{~B}_{4}$. Nanoscale $12,8314-8319$. doi: 10.1039/D0NR00635A

Wang, X., Ding, G., Khandy, S., Cheng, Z., Zhang, G., Wang, X., et al. (2020d). Unique topological nodal line states and associated exceptional thermoelectric power factor platform in $\mathrm{Nb}_{3} \mathrm{GeTe}_{6}$ monolayer and bulk. Nanoscale 12, 16910-16916. doi: 10.1039/D0NR03704D

Wang, X., Zhou, F., and Chen, H. (2020e). Organic-inorganic hybrid coordination polymer $\mathrm{C}_{3} \mathrm{H}_{9} \mathrm{CdCl}_{3} \mathrm{~N}$ co-exhibiting superior Dirac point and nodal surface states. Results Phys. 17:103159. doi: 10.1016/j.rinp.2020.103159

Wang, X. T., Ding, G., Cheng, Z. X., Surucu, G., and Yang, T. (2020a). Novel topological nodal lines and exotic drum-head-like surface states in synthesized CsCl-type binary alloy TiOs. J. Adv. Res. 22, 137-144. doi: 10.1016/j.jare.2019.12.001

Wang, X. T., Ding, G., Cheng, Z. X., Wang, X. L., and Yang, T. (2020b). Intersecting nodal rings in orthorhombic-type $\mathrm{BaLi}_{2} \mathrm{Sn}$ compound. J. Mater. Chem. C 8 , 5461-5466. doi: 10.1039/D0TC00504E

Weng, H., Fang, C., Fang, Z., and Dai, X. (2016a). Coexistence of Weyl fermion and massless triply degenerate nodal points. Phys. Rev. B 94:165201. doi: 10.1103/PhysRevB.94.165201

Weng, H., Fang, C., Fang, Z., and Dai, X. (2016b). Topological semimetals with triply degenerate nodal points in $\theta$-phase tantalum nitride. Phys. Rev. $B$ 93:241202. doi: 10.1103/PhysRevB.93.241202

Wu, M., Han, Y., Bouhemadou, A., Cheng, Z., and Wang, X. (2019). Site preference and tetragonal distortion in palladium-rich Heusler alloys. IUCrJ 6, 218-225. doi: $10.1107 /$ S2052252518017578

Wu, W., Liu, Y., Li, S., Zhong, C., Yu, Z. M., and Sheng, X. L. (2018). Nodal surface semimetals: Theory and material realization. Phys. Rev. B 97:115125. doi: 10.1103/PhysRevB.97.115125

Xia, Y., and Li, G. (2017). Triply degenerate nodal points and topological phase transitions in $\mathrm{NaCu}_{3} \mathrm{Te}_{2}$. Phys. Rev. B 96:241204 doi: 10.1103/PhysRevB.96.241204

Xiao, M., Ye, L., Qiu, C., He, H., Liu, Z., and Fan, S. (2020). Experimental demonstration of acoustic semimetal with topologically charged nodal surface. Sci. Adv. 6:eaav2360. doi: 10.1126/sciadv.aav2360

Xie, H., Qie, Y., Imran, M., and Sun, Q. (2019). Topological semimetal porous carbon as a high-performance anode for Li-ion batteries. J. Mater. Chem. A 7, 14253-14259. doi: 10.1039/C9TA03587G

Xu, S. G., Zheng, B., Xu, H., and Yang, X. B. (2019). Ideal nodal line semimetal in a two-dimensional boron bilayer. J. Phys. Chem. C 123, 4977-4983. doi: $10.1021 /$ acs.jpcc. 8 b12385

Yan, L., Liu, P. F., Bo, T., Zhang, J., Tang, M. H., and Xiao, Y. G. (2019). Emergence of superconductivity in a Dirac nodal-line $\mathrm{Cu}_{2} \mathrm{Si}$ monolayer: ab initio calculations. J. Mater. Chem. C 7, 10926-10932. doi: 10.1039/C9TC03740C

Yan, Q., Liu, R., Yan, Z., Liu, B., Chen, H., Wang, Z., et al. (2018). Experimental discovery of nodal chains. Nat. Phys. 14, 461-464. doi: 10.1038/s41567-017-0041-4

Yan, Z., Bi, R., Shen, H., Lu, L., Zhang, S. C., and Wang, Z. (2017). Nodal-link semimetals. Phys. Rev. B 96:041103. doi: 10.1103/PhysRevB.96.041103

Yang, T., Khenata, R., and Wang, X. (2020). Predicted remarkably topological nodal surface states in $\mathrm{P}_{3} / \mathrm{m}$ type $\mathrm{Sr}_{3} \mathrm{WN}_{3}$ from first-principles. Results Phys. 17:103026. doi: 10.1016/j.rinp.2020.103026

Yang, T., and Zhang, X. (2020). Nearly flat nodal surface states in pseudoone-dimensional molybdenum monochalcogenides $\mathrm{X}(\mathrm{MoS})_{3} \quad(\mathrm{X}=$ $\mathrm{K}, \mathrm{Rb}$, and Cs). J. Mater. Chem. C 8, 9046-9054. doi: 10.1039/D0TC 01978J

Yang, Y., Sun, H. X., Xia, J. P., Xue, H., Gao, Z., Ge, Y., et al. (2019a). Topological triply degenerate point with double Fermi arcs. Nat. Phys. 15, 645-649. doi: 10.1038/s41567-019-0502-z

Yang, Y., Xia, J. P., Sun, H. X., Ge, Y., and Zhang, B. (2019b). Observation of a topological nodal surface and its surface-state arcs in an artificial acoustic crystal. Nat. Commun. 10, 1-7. doi: 10.1038/s41467-019-13258-3

Yi, X., Li, W., Li, Z., Zjou, P., Ma, Z., and Sun, L. (2019). Topological dual double node-line semimetals $\mathrm{NaAlSi}(\mathrm{Ge})$ and their potential as cathode material for sodium ion batteries. J. Mater. Chem. C 7, 15375-15381. doi: 10.1039/C9TC04096J 
Yu, R., Weng, H., Fang, Z., Dai, X., and Hu, X. (2015). Topological node-line semimetal and Dirac semimetal state in antiperovskite $\mathrm{Cu}_{3} \mathrm{PdN}$. Phys. Rev. Lett. 115:036807. doi: 10.1103/PhysRevLett.115.036807

Yuan, X., Cheng, P., Zhang, L., Zhang, C., and Xiu, F. (2017). Direct observation of Landau level resonance and mass generation in dirac semimetal $\mathrm{Cd}_{3} \mathrm{As}_{2}$ thin films. Nano Lett. 17, 2211-2219. doi: 10.1021/acs.nanolett.6b04778

Zhang, C., Zhang, E., Wang, W., Liu, Y., Chen, Z. G., and Lu, S. (2017a). Roomtemperature chiral charge pumping in dirac semimetals. Nat. Commun. 8, 1-9. doi: $10.1038 /$ ncomms 13741

Zhang, L., Zhang, S., Ji, W., Zhang, C., Li, P., Wang, P., et al. (2018a). Discovery of a novel spin-polarized nodal ring in a two-dimensional HK lattice. Nanoscale 10, 20748-20753. doi: 10.1039/C8NR05383A

Zhang, M. H., Zhang, S. F., Wang, P. J., and Zhang, C. W. (2020). Emergence of a spin-valley Dirac semimetal in a strained group-VA monolayer. Nanoscale 12, 3950-3957. doi: 10.1039/C9NR09545D

Zhang, T. T., Yu, Z. M., Guo, W., Shi, D., Zhang, G., and Yao, Y. (2017b). From type-II triply degenerate nodal points and three-band nodal rings to typeII Dirac points in centrosymmetric zirconium oxide. J. Phys. Chem. Lett. 8, 5792-5797. doi: 10.1021/acs.jpclett.7b02642

Zhang, X., Fu, B., Jin, L., Dai, X., and Yao, Y. (2019). Topological nodal line electrides: realization of an ideal nodal line state nearly immune from spin-orbit coupling. J. Phys. Chem. C 123, 25871-25876. doi: 10.1021/acs.jpcc.9b08446

Zhang, X., Jin, L., Dai, X., and Liu, G. (2017c). Topological type-II nodal line semimetal and Dirac semimetal state in stable kagome compound $\mathrm{Mg}_{3} \mathrm{Bi}_{2}$. J. Phys. Chem. Lett. 8, 4814-4819. doi: 10.1021/acs.jpclett.7b02129

Zhang, X., Jin, L., Dai, X., and Liu, G. (2018b). Highly anisotropic typeII nodal line state in pure titanium metal. Appl. Phys. Lett. 112:122403. doi: $10.1063 / 1.5023320$

Zhang, X., Liu, Q., Xu, Q., Dai, X., and Zunger, A. (2018c). Topological insulators versus topological Dirac semimetals in honeycomb compounds. J. Am. Chem. Soc. 140, 13687-13694. doi: 10.1021/jacs.8b06652

Zhang, X., Yu, Z., Sheng, X., Yang, H., and Yang, S. A. (2017d). Coexistence of four-band nodal rings and triply degenerate nodal points in centrosymmetric metal diborides. Phys. Rev. B 95:235116. doi: 10.1103/PhysRevB.95. 235116
Zhang, X., Yu, Z. M., Zhu, Z., Wu, W., and Yang, S. A. (2018d). Nodal loop and nodal surface states in the Ti 3 Al family of materials. Phys. Rev. B 97:235150. doi: 10.1103/PhysRevB.97.235150

Zhao, M., Dong, W., and Wang, A. (2013). Two-dimensional carbon topological insulators superior to graphene. Sci. Rep. 3:3532. doi: 10.1038/srep03532

Zhao, Z., Zhang, Z., and Guo, W. (2020). A family of all sp 2-bonded carbon allotropes of topological semimetals with strain-robust nodal-lines. J. Mater. Chem. C 8, 1548-1555. doi: 10.1039/C9TC05470G

Zhong, C., Wu, W., He, J., Ding, G., Liu, Y., and Li, D. (2019). Twodimensional honeycomb borophene oxide: strong anisotropy and nodal loop transformation. Nanoscale 11, 2468-2475. doi: 10.1039/C8NR08729F

Zhou, P., Ma, Z., and Sun, L. (2018). Coexistence of open and closed type nodal line topological semimetals in two dimensional $\mathrm{B}_{2}$ C. J. Mater. Chem. C 6 , 1206-1214. doi: 10.1039/C7TC05095J

Zhou, Q., Wang, J., Chwee, T. S., Wu, G., Wang, X., and Ye, Q. (2015). Topological insulators based on 2D shape-persistent organic ligand complexes. Nanoscale 7 , 727-735. doi: 10.1039/C4NR05247A

Zhou, T., Tong, M., Xie, X., Yu, Y., Zhu, X., Wang, Z. Y., et al. (2020). Quantum transport signatures of a close candidate for a type II nodal-line semimetal. J. Phys. Chem. Lett. 11, 6475-6481. doi: 10.1021/acs.jpclett.0c01726

Zhu, Y., Gui, X., Wang, Y., Graf, D., Xie, W., Hu, J., et al. (2020). Evidence from transport measurements for $\mathrm{YRh}_{6} \mathrm{Ge}_{4}$ being a triply degenerate nodal semimetal. Phys. Rev. B 101:035133. doi: 10.1103/PhysRevB.101.035133

Conflict of Interest: The author declares that the research was conducted in the absence of any commercial or financial relationships that could be construed as a potential conflict of interest.

The handling editor declared a past co-authorship with one of the authors, HX.

Copyright (c) $2020 \mathrm{Xu}$. This is an open-access article distributed under the terms of the Creative Commons Attribution License (CC BY). The use, distribution or reproduction in other forums is permitted, provided the original author(s) and the copyright owner(s) are credited and that the original publication in this journal is cited, in accordance with accepted academic practice. No use, distribution or reproduction is permitted which does not comply with these terms. 